\title{
Improvement in clinical recording keeping following the introduction of an admission clerking proforma for acute general surgical patients
}

\author{
This article was published in the following Dove Press journal: \\ Clinical Audit \\ 13 August 2013 \\ Number of times this article has been viewed
}

\section{Shradha Gupta \\ Kumaran Ratnasingham \\ Chi Tamura \\ Ravindran Karthigan \\ Veena Bhargava \\ Nicholas West \\ General Surgery Department, St Helier Hospital, Epsom and St Helier University Hospitals, Carshalton, UK}

Correspondence: Shradha Gupta C/- Nicholas West, General Surgery Department, St Helier Hospital,

Carshalton, UK

Tel +440795I726648

Email shrada.gupta@nhs.net
Background: In 2008, the Royal College of Physicians (RCP) alongside National Health Service Connecting for Health endorsed standards for patient records to improve patient safety by standardizing the information held on patients throughout their stay in hospital. Opinion on accurate recordkeeping, safe handover, and optimal management of acute surgical patients has reached a consensus within general surgical practice since the publication of the Handover Guidance and the Emergency Surgery Standards by the Royal College of Surgeons of England. This audit assesses the improvement in clerking notes following implementation of a proforma for acute surgical admissions.

Method: The surgical admission clerking notes of 100 acute patients were audited against standards derived from the Handover Guidance and Emergency Surgery Standards, and RCP Record Keeping Standards. A standardized proforma was constructed and implemented across the unit and 100 patient notes were audited in a second audit cycle.

Results: The proforma significantly improved documentation across multiple fields including patient history, patient examination, and investigations $(P<0.05)$. Completion of venous thromboembolism risk assessment increased by $62 \%(P<0.001)$. There was increased documentation of the time taken until senior review of the patient post-admission, which occurred within an average of 323 minutes, an overall improvement of 173 minutes.

Conclusion: The use of a surgical clerking proforma on admission has been shown to improve documentation significantly, and standardize the information recorded for patients admitted in an acute general surgical setting. A proforma can also be used as an audit tool to measure against national standards.

Keywords: safe handover, acute surgery, proforma, patient safety, recordkeeping

\section{Introduction}

Patient records are a vital means of communication between health care providers. Following the introduction of the working time directive there have been changes in the working practices of doctors with the introduction of shift systems and increased transfer of responsibility between individuals and teams. Accurate documentation has become key to minimize error and optimize patient safety. Clear documentation benefits the health care professional as it provides a means of examining the decisionmaking processes governing patient management. Documentation is also an important legal document that may need to be relied upon in future court disputes and medical tribunals, so that clarity and accuracy are paramount. 
In 2008, the Royal College of Physicians in partnership with NHS Connecting for Health endorsed a set of standards ${ }^{1}$ for maintaining hospital patient records to improve safety by standardizing patient notes throughout their stay in hospital. Additionally, in 2007, the Royal College of Surgeons of England published their document on Safe Handover Guidance $^{2}$ promoting safe patient handover. In 2011, they developed The Emergency Surgery Standards ${ }^{3}$ setting out important objectives specifically directed towards optimizing the management of acute surgical patients admitted into hospital in order to deliver safer and more efficient care and to improve patient outcome.

We formulated a surgical admission proforma, novel within our Trust, based on these standards, and audited its effect on improving the adequacy and consistency of record keeping alongside improving the management of acute general surgical admissions.

\section{Method}

A prospective two-cycle audit was conducted between October 2011 and February 2012, examining the completion of admission clerking documentation for acute general surgical patients presenting at a district general hospital.

A paper proforma was constructed to encompass the standards of excellence set out in the Royal College of Surgeons' Handover Guidance, the required standards upon admission for general surgical patients set out in the Royal College of Surgeons Emergency Surgery Standards, and the Royal College of Physicians Record Keeping Standards. ${ }^{1-3}$

In combination, the standards set out in these documents state that all notes must include:

- Patient name

- Patient age and date of birth

- Hospital identification number

- Admitting doctor's details including name, grade, contact number/pager

- Responsible consultant in charge

- Full history, examination, and initial observations

- Results of investigations or pending investigations

- Management plan

- Resuscitation plan if appropriate

- Documentation of venous thromboembolism risk assessment

- Each entry dated and timed with the name, designation, and contact details of the doctor performing the entry.
In addition, the Emergency Standards specify that to comply with good practice:

- All patients should be discussed with the consultant within 12 hours

- The consultant must see all patients within 24 hours.

In the first cycle, 100 consecutive patient notes were collected and audited. After the daily post-take ward round, the admission clerking notes were checked against the sample proforma for the required information using a "tick" or "cross" to demonstrate completed and incomplete information respectively. The date and time of the initial clerking was recorded as was the date and time of the initial senior review. The specific investigations that were originally requested were compared with the investigations with documented results.

Using the set standards (as described earlier), the emergency surgical clerking proforma was developed by the authors. The compulsory standards for admission notes were printed on subsequent pages as guidance headings and subheadings with adequate space for the admitting doctor to document the required information. The booklets were dispersed throughout the department including all the acute general surgery wards, and Accident and Emergency. Members of the general surgical team were educated about the new initiative in departmental meetings. Results of the first audit cycle were concealed in order to minimize any bias when reauditing the data.

One month after introduction, the admission booklets for a further 100 consecutive patients were audited against the same standards. The same author performed the data collection in both cycles using the same method of data collection. The results for document completion were statistically analyzed using a Chi-squared test; the significance level was taken as $P<0.05$. A simple $t$-test was applied to compare times to senior review.

\section{Results}

Figure 1 compares the completion of patient information, the details of the clerking doctor, and leading health care provider (ie, on-call consultant) between the first (pre-proforma introduction) and second (post-proforma introduction) audit cycles. After the introduction of the proforma, there was a significant increase in documentation of the on-call consultant, leading patient care $(P<0.001,95 \%$ CI, 80.4-94.4); completion of patient number $(P=0.019,95 \% \mathrm{CI}, 1.9-18.5)$; and completion of doctor name ( $P<0.001,95 \% \mathrm{CI}, 6.9-22.4)$.

Figure 2 compares the completeness of patient history. There was a significant improvement in recording the patient's past medical history $(P=0.02,95 \% \mathrm{CI}, 1.5-13.9)$; social history $(P=0.006, \mathrm{CI}, 4.1-23.9)$; drug history $(P=0.0131$, 


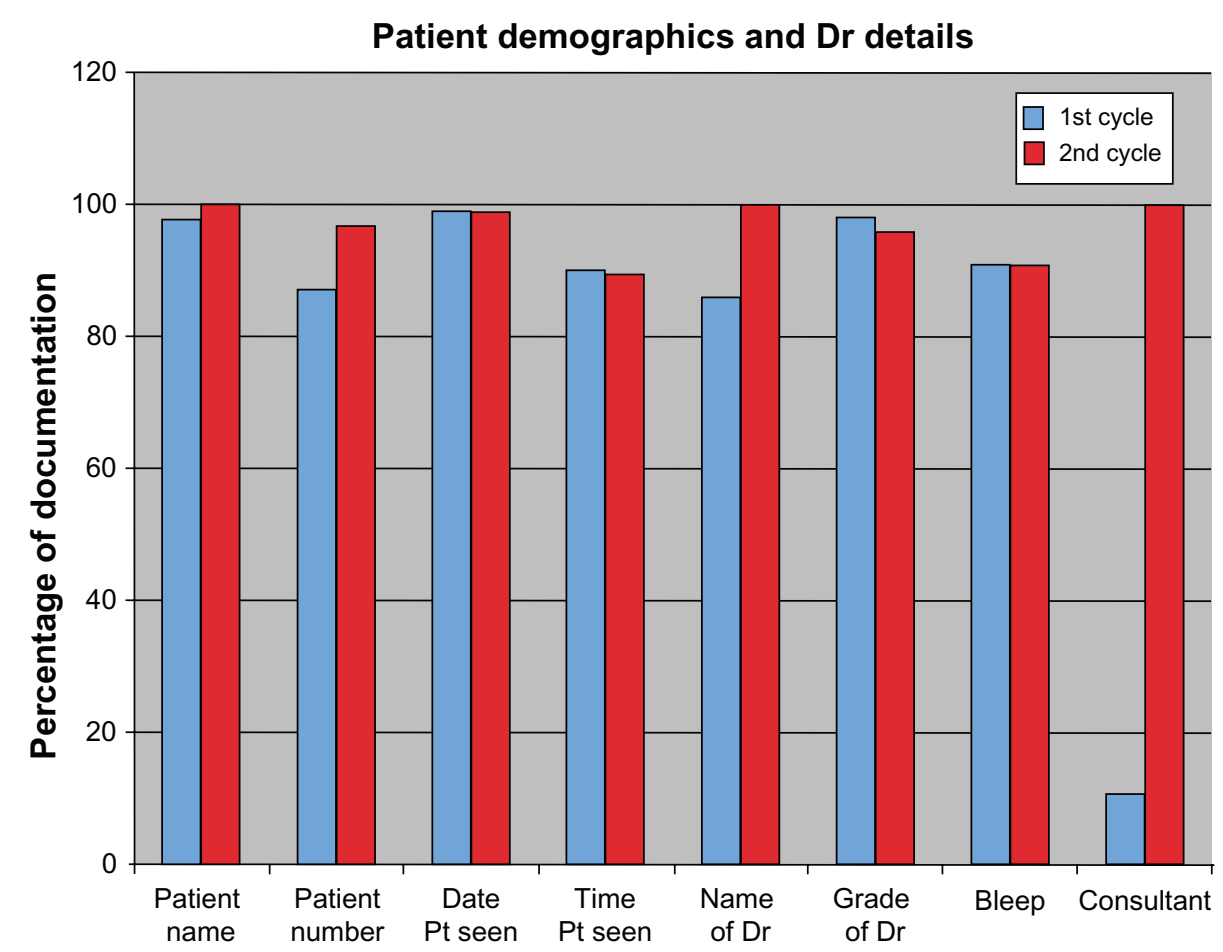

Figure I Comparison of the accuracy in documenting patient information, details of the clerking doctor, and leading health care provider. Note: $\mathrm{n}=100$.

Abbreviations: Pt, patient; Dr, clerking doctor between first and second audit cycle.

95\% CI, 2.2-16.7; alcohol consumption $(P<0.001,95 \%$ CI, 17.2-40.2); smoking history $(P<0.001,95 \% \mathrm{CI}, 18.5-40.9)$; and family history $(P<0.001,95 \% \mathrm{CI}, 55.7-77.3)$.

Figure 3 compares the accuracy of documenting a full patient examination including respiratory, cardiovascular and abdominal examination; patient observations at examination; investigations ordered and their results and the subsequent management plan. It includes whether a venous thromboembolism risk assessment was documented in the proforma. This acted as a reminder to the admitting doctor as venous thromboembolic risk assessments are mandatorily entered onto the Trust computer system.

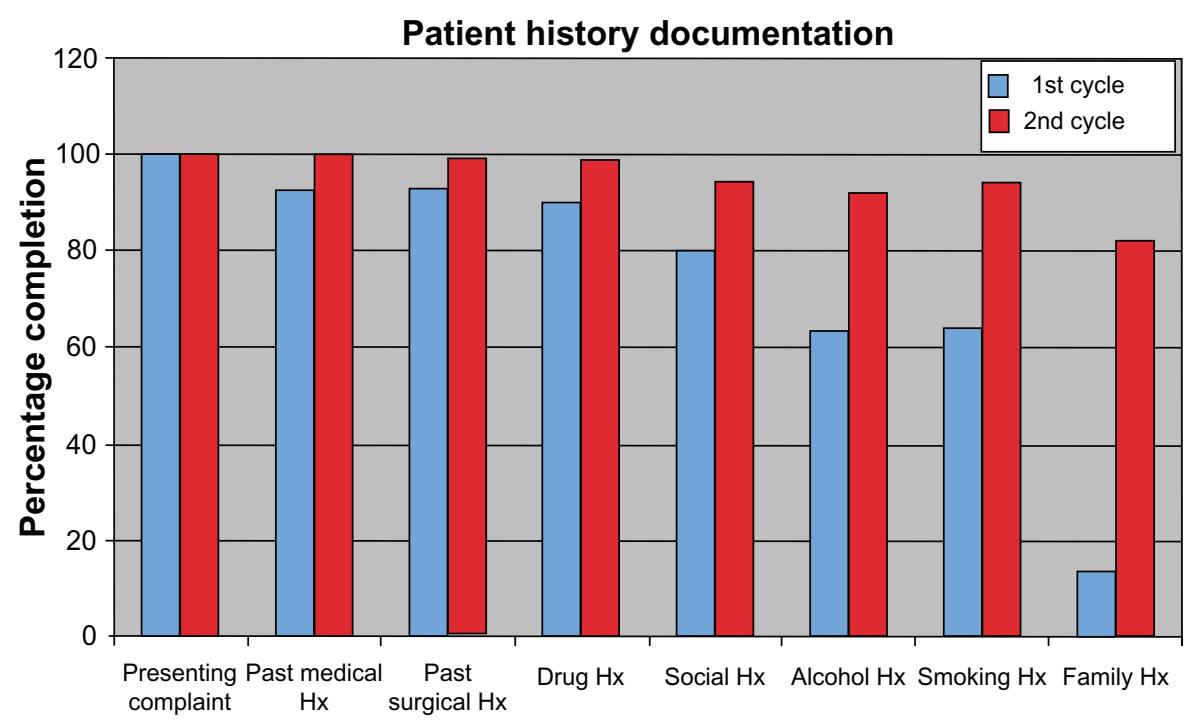

Figure 2 Comparison of the completeness of patient history between first and second audit cycle.

Note: $\mathrm{n}=100$

Abbreviation: $\mathrm{Hx}$, history. 


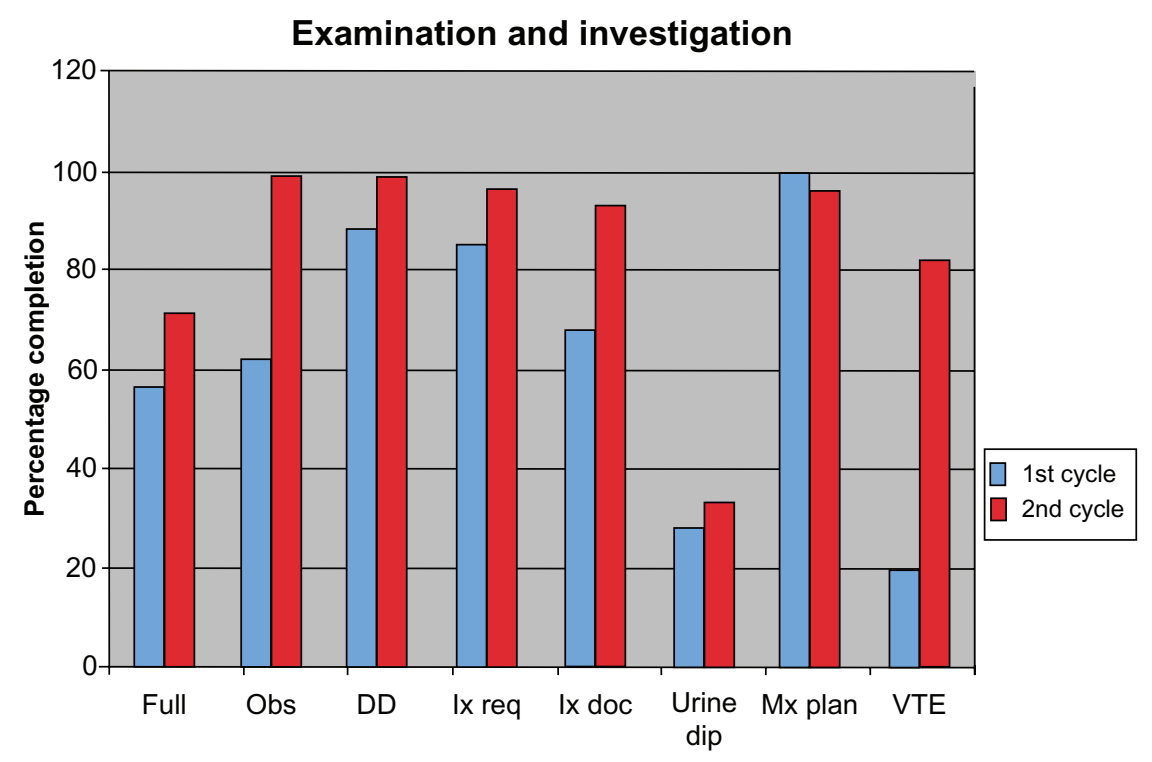

Figure 3 Comparison of the accuracy of documenting a full patient examination including respiratory, cardiovascular, and abdominal examination (Full); patient observations at examination (Obs); differential diagnosis (DD) investigations requested (Ix req); investigations documented (Ix doc); urine dip; and subsequent management plan (Mx plan). It includes whether a venous thromboembolism risk assessment was completed for the patient (VTE) between first and second audit cycle.

Note: $n=100$.

Performing a complete patient examination by the clerking doctor improved significantly $(P=0.027,95 \%$ CI, 2-29.4). There was a significant improvement in the documentation of observations $(P<0.001,95 \% \mathrm{CI}$, 26.5-47.3); ordered investigations $(P=0.0066,95 \% \mathrm{CI}$, 3.6-20.9); results of ordered investigations $(P<0.001$, 95\% CI 13.7-35.9); differential diagnosis ( $P=0.007,95 \%$ CI, 3-17.9). There was a significant increase in completing the venous thromboembolism risk assessment, $(P<0.001$, 95\% CI, 49.2-72.1).

Table 1 describes the time taken for initial review of the patient by a senior doctor, defined as Specialist Trainee Level 3 (ST3) and above, following admission between first and second audit cycle. There was a significant improvement in the documentation of the date of initial senior review $(P=0.02,95 \% \mathrm{CI}, 1.6-17)$ and time of the initial senior review after the introduction of the proforma $(P=0.04$, $95 \% \mathrm{CI}, 0.7-23.1)$. The mean time until senior review was

Table I The proportion of completed documentation for the initial date and time of patient review by a senior doctor, defined as Specialist Trainee Level 3 (ST3) and above, following admission between first and second audit cycle

\begin{tabular}{lll}
\hline Documented data & $\begin{array}{l}\text { Ist audit } \\
\text { cycle (\%) }\end{array}$ & $\begin{array}{l}\text { 2nd audit } \\
\text { cycle (\%) }\end{array}$ \\
\hline $\begin{array}{l}\text { Documented date of initial } \\
\text { senior review (ST3+) }\end{array}$ & 89 & 98 \\
$\begin{array}{l}\text { Documented time of initial } \\
\text { senior review (ST3+) }\end{array}$ & 76 & 88 \\
\hline
\end{tabular}

496 minutes (standard deviation $=1.1$ ) in cycle one and this improved significantly $(P<0.001,95 \% \mathrm{CI},-2.8$ to -2.2$)$ to 323 minutes in cycle two (standard deviation $=0.8$ ).

Table 2 offers a further breakdown of the grade of senior doctor reviewing the patient between first and second audit cycle.

\section{Discussion}

Employing a standardized surgical clerking proforma significantly improved overall patient documentation for acute surgical admissions.

In this audit, the introduction of the clerking booklet increased documentation of patient and doctor details to above $90 \%$. There was a significant improvement in completion of the patient history including the social history, family history, and the patient's alcohol and smoking consumption.

The admitting doctors documented a greater number of full patient examinations as opposed to, eg, just an abdominal examination. The documentation of initial observations and investigation results also improved.

Table 2 Further breakdown of the grade of senior doctor reviewing the patient between first and second audit cycle

\begin{tabular}{lll}
\hline $\begin{array}{l}\text { Grade of senior } \\
\text { doctor }\end{array}$ & $\begin{array}{l}\text { Ist audit } \\
\text { cycle (\%) }\end{array}$ & $\begin{array}{l}\text { 2nd audit } \\
\text { cycle (\%) }\end{array}$ \\
\hline Consultant & 23 & 42 \\
ST3+ & 75 & 53 \\
No senior review & 2 & 5 \\
\hline
\end{tabular}

Abbreviation: ST3, Specialist Trainee Level 3. 
The proforma acts as a template, by inclusion of relevant headings, and therefore seems to prompt the admitting doctor to perform and document these details. The process of history taking varies between doctors as each develops their unique style of clerking, which is acquired in an apprenticeship style and molded by experience, so that the structure and content of notes may vary. The proforma serves as a formatted aidememoire for the admitting doctor to ensure that important details are not overlooked. This audit has demonstrated that by using a proforma, information is more likely to be recorded adequately and consistently for every patient, minimizing variation in quality.

There was a reduction in the documentation of a management plan as four patients were missing a clear management plan in their notes; unfortunately there was no obvious explanation for these omissions and it did not correspond with inadequate completion of other areas of the booklet. Ongoing departmental education reiterating the proper completion of the clerking booklet is required periodically.

In accordance with the Emergency Surgery Standards ${ }^{3}$ all patients should have a venous thromboembolism risk assessment on admission. This practice is in keeping with national guidance ${ }^{4}$ and is being enforced across trusts, subsequent to the 2005 House of Commons Health Committee report ${ }^{5}$ that an estimated 25,000 people in the UK die from preventable hospital-acquired venous thromboembolism every year. This practice is regularly being audited across hospitals in the $\mathrm{UK}^{6-8}$ and is an essential component of clinical governance. Inclusion of the venous thromboembolism risk assessment within the proforma significantly $(P<0.001)$, improved its completion on admission

The Emergency Standards maintain that surgical patients, who are not listed for an immediate operation, should be discussed with the responsible consultant within 12 hours of admission and a consultant surgeon should review them personally within 24 hours of admission. Senior review by ST3 and above within 12 hours facilitates subsequent discussion with the consultant. The average time between admission and a senior review by a specialist trainee doctor was reduced in the second cycle. There was also an increase in the documented number of consultant-led reviews within 24 hours from $23 \%$ to $42 \%$. Early consultant review is important and has been recognized by the Royal College of Surgeons to prevent inappropriate delays in patient care, which could place the patient at increased risk of an adverse outcome. It is difficult to explain why the proforma should reduce the time taken for senior review and increase consultant-led reviews. It is likely that the date and time of the patient's admission, subsequent senior review, and consultant-led review were all better documented, as opposed to an actual change in practice. However, the results show that further improvement is required as more than $50 \%$ of patients were not reviewed by a consultant within 24 hours. This documentation is in itself vital because it is the only legal record of the management a patient has received. It is not only important for patient safety and providing accountability for the decisions made, but is also a safety-net for the doctors as proof that reasonable care has been delivered.

Clerking proformas have been trialed within other specialties. Diggory et al ${ }^{9}$ found that after introducing a clerking proforma, documentation of cardiopulmonary resuscitation decisions improved from $36 \%$ to $94 \%$. Wethers and Brown ${ }^{10}$ implemented a clerking proforma for patients admitted onto a general psychiatric ward. They found that it significantly improved the completeness of clerking especially "in multiple components of history taking and initial management." Our study records the first time that a clerking proforma has been applied for acute general surgical patients.

The proforma directs the doctor to complete all necessary information. It collects this information in a structured format, which can be easily referenced when the patient is being reviewed. Because the proforma, is accessible to all members of the multidisciplinary team, it can facilitate their management plans. The standardized format communicates the decision-making process of the admitting doctor in a logical manner that has potential to be used as a learning adjunct for junior and senior doctors.

There are many limitations to this audit study. The assessor bias cannot be improved, as it was not a blinded study. It is difficult to rule out confounding factors, as it was difficult to ensure that all other external factors were kept the same in both cycles. One can argue that when something like a proforma is introduced, it is utilized better in the early period. Therefore it will be useful to repeat this audit in 6 months time to validate that the improvements are maintained.

\section{Conclusion}

Employing a surgical clerking proforma has been shown to significantly improve the completion of documentation for patients admitted acutely under the general surgical team. It can be used to reaudit future delivery of care against the standards that have been proposed by the Royal College of Surgeons and the Royal College of Physicians as well as function as a learning tool to improve practices within the multidisciplinary team and consequently improve overall patient care. 


\section{Disclosure}

There were no external contributors. The authors have no conflicts of interest to declare in this work. No funding was required for this audit.

\section{References}

1. United Kingdom. A Clinician's Guide to Record Standards - Part 2: Standards for the structure and content of medical records and communications when patients are admitted to hospital. London: Department of Health; 2008.

2. The Royal College of Surgeons of England Safe Handover: Guidance from the Working Time Directive working party: The Royal College of Surgeons United Kingdom; 2007.

3. Emergency Surgery Standards for unscheduled surgical care: Guidance for providers, commissioners and service planners. Royal College of Surgeons of England; 2011.

4. United Kingdom. National Institute for Health and Clinical Excellence. Venous thromboembolism: reducing the risk: Reducing the risk of venous thromboembolism (deep vein thrombosis and pulmonary embolism) in patients admitted to hospital. Clinical Guidelines CG92; Jan 2010.
5. United Kingdom. House of Commons Health Committee. The Prevention of Venous Thromboembolism in Hospitalised Patients. London: The Stationery Office; 2005.

6. Veeramootoo D, Harrower L, Saunders R, et al. Prophylaxis of venous thromboembolism in general surgery: guidelines differ and we still need local policies. Ann R Coll Surg Engl. 2011;93(5):370-374.

7. Sadideen H, O'Callaghan JM, Navidi M, et al. Educating surgical patients to reduce the risk of venous thromboembolism: an audit of an effective strategy. JRSM Short Rep. 2011;2(12):97.

8. Vaughan-Shaw PG, Cannon C. Venous thromboembolism prevention in medical patients: a framework for improving practice. Phlebology. 2011;26(2):62-68.

9. Diggory P, Shire L, Griffith D, et al. Influence of guidelines on CPR decisions: an audit of clerking proforma. Clin Med. 2004;4(5): 424-426.

10. Wethers G, Brown J. Does an admission booklet improve patient safety? J Ment Health. 2011;20(5):438-444.
Clinical Audit

\section{Publish your work in this journal}

Clinical Audit is an international, peer-reviewed, open access journal focusing on the processes and outcomes of clinical audit in any area of healthcare. All aspects of patient care are addressed within the journal and practitioners from all disciplines are invited to submit their work. Areas covered include: Publication of audits; How an audit has changed practice;

\footnotetext{
Submit your manuscript here: http://www.dovepress.com/clinical-audit-journal
}

\section{Dovepress}

Practical tips on how to do audits and to avoid pitfalls; How audits have changed patient care; Calls and justifications for new audits. The manuscript management system is completely online and includes a very quick and fair peer-review system, which is all easy to use. Visit http://www.dovepress. com/testimonials.php to read real quotes from published authors. 